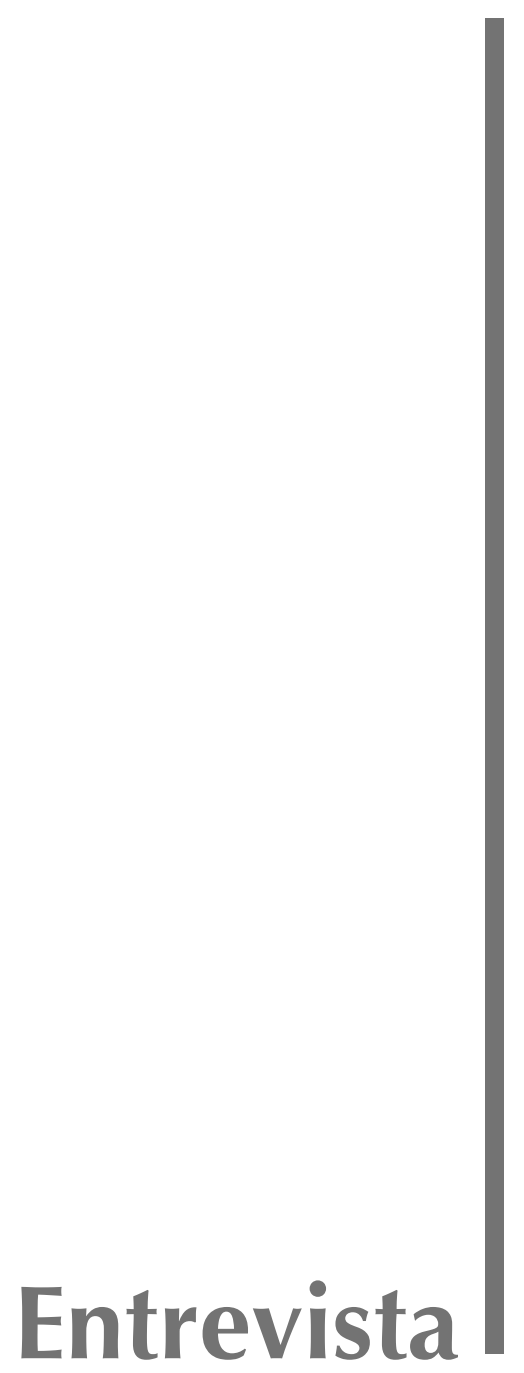





\title{
Entrevista a Rebecca Cook: “Estereotipos de Género: Perspectivas Legales Transnacionales"**
}

\author{
Por Nicole Lacrampette
}

Nicole Lacrampette es abogada, Licenciada en Ciencias Jurídicas y Sociales (2011) y BachiIler en Humanidades y Ciencias Sociales (2004) por la Universidad de Chile. Trabajó como investigadora del Programa "Mujeres, Género y Derechos Humanos" del Centro de Derechos Humanos de la Facultad de Derecho de la Universidad de Chile y como editora de la Revista Electrónica "Género, Sexualidades y Derechos Humanos". Ha colaborado con Women's Link Worldwide a través de su programa de prácticas y actualmente es becaria en la Relatoría de Derechos de las Mujeres de la Comisión Interamericana de Derechos Humanos.

Rebecca Cook es profesora emérita y codirectora del Programa Internacional de Derecho en Salud Sexual y Reproductiva de la Facultad de Derecho de la Universidad de Toronto. Es LL.M y J.S.D de la Universidad de Columbia. Es profesora en la Facultad de Derecho, la Facultad de Medicina y el Centro Asociado de Bioética de la Universidad de Toronto. Es coeditora en asuntos legales y éticos de la revista International Journal of Gynecology and Obstetrics y forma parte de los consejos asesores de las publicaciones Human Rights Quarterly y Reproductive Health Matters. Ha sido distinguida con el Certificate of Recognition for Outstanding Contribution to Women's Health otorgado por la International Federation of Gynecologists and Obstetricians, así como con el premio Ludwik and Estelle Jus Memorial Human Rights Prize. Cuenta con diversas publicaciones en el área de la salud sexual y reproductiva de las mujeres, entre ellas el libro Gender Stereotyping: Transnational Legal Perspectives, traducido al español como Estereotipos de Género: Perspectivas Legales Transnacionales.

\section{Introducción}

Un estereotipo, en general, es una visión generalizada o una preconcepción sobre los atributos o características de los miembros de un grupo en particular o sobre los roles que tales miembros deben cumplir. Es decir, la particularidad de los estereotipos es que se aplican a todos los miembros de un determinado grupo con prescindencia de las características específicas de cada individuo.

Desde la psicología se ha planteado que los estereotipos pueden conceptualizarse desde dos perspectivas complementarias: por una parte, los estereotipos se representan en la mente de la persona individual, y por otra, los estereotipos se representan como parte del tejido social de las sociedades y son compartidos por las personas dentro de una cultura ${ }^{1}$. El proceso de asignar estereotipos, en este sentido, es parte de la naturaleza humana y cumple con funciones concretas. Una de las funciones más básicas de los estereotipos es proporcionar información útil acerca de los otros, es decir, los estereotipos operan de manera tal que permiten a los individuos utilizar sus creencias acerca de los grupos sociales para lograr entender y explicar los comportamientos individuales ${ }^{2}$.

* Entrevista traducida del inglés por Nicole Lacrampette.

STANGOR, Charles y SCHALLER, Mark. "Stereotypes as individual and collective representations". En: MACRAE, C. Neil, STANGOR, Charles y HEWSTONE, Miles. Stereotypes and stereotyping. New York: The Guilford Press, 1996, p. 4.

Ibídem, pp. 20 y ss. 
Los estereotipos de género -aquellos referidos a la construcción social y cultural de hombres y mujeres, en razón de sus diferentes funciones físicas, biológicas, sexuales y sociales- suelen encontrarse en la base de muchos de los casos de discriminación que afectan a las mujeres. En el libro "Estereotipos de Género: Perspectivas Legales Transnacionales" de Rebecca J. Cook y Simone Cusack $^{3}$, se analiza en profundidad la relación entre la estereotipación de género y la discriminación contra las mujeres, y se propone una metodología para identificar la presencia de tales estereotipos en las leyes, políticas públicas y prácticas. Con ello se busca visibilizar las preconcepciones sobre las mujeres que determinan que sean tratadas de forma desigual y la forma en que esos prejuicios se encuentran institucionalizados en la sociedad a través de las leyes, políticas públicas y decisiones judiciales. De esta forma, la identificación de los estereotipos de género se presenta como una herramienta para desarticular las desigualdades de género que provienen de las preconcepciones individuales y colectivas acerca de las capacidades y roles de las mujeres y que suelen estar invisibilizadas.

En esta entrevista, la profesora Rebecca Cook -una de las coautoras del libro referido- se refiere al uso de los estereotipos de género como una forma de combatir la discriminación desde el Derecho, comentando acerca de los principales avances en esta materia en el ámbito del Derecho Internacional de los Derechos Humanos y los desafíos que se prevén a futuro.

\section{Nicole Lacrampette: ¿De qué forma los estereotipos de género perjudican a las mujeres?}

Rebecca Cook: Los estereotipos de género perjudican tanto a hombres como a mujeres, en el sentido de que frecuentemente pueden ocasionar que se les nieguen beneficios tales como ascensos en el empleo e incluso ser contratados/as. Un ejemplo es el caso de una mujer a quien se niega el ascenso a un cargo de liderazgo o la participación social en una empresa de contabilidad, o el caso de un hombre a quien se niega la admisión para estudiar enfermería. Es así como, frecuentemente, los estereotipos operan de forma discriminatoria, negando ciertos beneficios a hombres y mujeres.

Los estereotipos de género también pueden ser perjudiciales en el sentido de imponer una carga a hombres o mujeres. Ello ocurre, por ejemplo, en los casos en que a las mujeres se les impone la obligación de permanecer en el hogar o los casos en que se les niega un juicio justo, como puede ocurrir en los casos de violación sexual. Estas son algunas de las formas en que los estereotipos de género pueden resultar perjudiciales.

Nicole Lacrampette: En el libro se sostiene que el estereotipar es parte de la naturaleza humana. Atendiendo a ello, ¿es realmente posible eliminar los estereotipos de género? Y si lo es, ¿no sería más efectivo apuntar los esfuerzos hacia la modificación de las construcciones sociales del género que resultan perjudiciales para las mujeres?

Rebecca Cook: Me parece que es posible modificar los estereotipos de género, pero debe hacerse con mucho cuidado y muy estratégicamente, comprendiendo el contexto en el que surgen los estereotipos. Por ejemplo, la estereotipación en el ámbito del empleo puede perpetuarse porque los empleadores aplican y reproducen estereotipos de género en sus evaluaciones de los empleados. Por ejemplo, un empleador podría decir que las mujeres no son lo suficientemente dominantes y en base a ello negarles un ascenso.

Pero creo que es muy importante comprender el contexto en el que esto surge. La mayor parte de lo que sabemos acerca de la estereotipación en la esfera del empleo se debe a una impre-

COOK, Rebecca y CUSACK, Simone. Estereotipos de Género: Perspectivas Legales Transnacionales. Bogotá: Profamilia, 2010 (Traducción de Andrea Parra). Título original: Gender Stereotyping: Transnational Legal Perspectives. University of Pennsylvania Press, 2009. 
sionante investigación en el campo de la psicología social. Sabemos algunas cosas acerca de la estereotipación en el ámbito de la educación, donde las niñas son dirigidas hacia las clases de economía doméstica y los niños hacia la carpintería, por ejemplo. Pero más allá de esto no tenemos mucho conocimiento acerca de los estereotipos de género en otros sectores y por ello es necesario más trabajo y más investigación para comprender la naturaleza de la estereotipación, el contexto en el que surge y la forma en que se perpetúa en diversos sectores, a fin de trabajar apropiadamente para eliminarla.

Ahora, respecto a la segunda pregunta, me parece que la comprensión de los estereotipos y prejuicios es una forma de desafiar las construcciones sociales del género. Los estereotipos son una forma de construir el género y creo que usar la psicología social para entender cómo son estereotipadas las mujeres y cómo son estereotipados los hombres, nos ayuda a desafiar estas construcciones sociales.

El libro se enfoca en cómo la ley puede eliminar la estereotipación de género, pero hay también otras vías. Por ejemplo, existen películas excelentes sobre este tema, en el libro citamos algunas como "Bending it like Beckham" que es una comedia; también citamos "A league of their own"4, en el que las mujeres quieren jugar baseball. Otra película que he visto recientemente y que es muy conmovedora, es de un director de Arabia Saudita y se trata de una adolescente de Arabia Saudita que quiere comprar una bicicleta. Se llama "Wadjda" y está realmente muy bien realizada, cuenta la historia de cómo esta joven trata de reunir el dinero para comprar una bicicleta y todas las fuerzas que están en su contra en la sociedad saudita, debido a que las mujeres normalmente no usan bicicletas en esa parte del mundo.

Entonces, la ley no es la única vía para identificar, nombrar y analizar la estereotipación. Se hace también en la literatura y en el cine, puede hacerse también con humor.

\section{Nicole Lacrampette: ¿Cuál es su opinión acerca de la forma en que el Comité CEDAW ha abordado la estereotipación de género en sus decisiones sobre casos en que ha declarado vio- laciones a los artículos 2.f) y 5.a) de la Convención? ¿Cuáles diría que son los principales retos para el Comité en este asunto?}

Rebecca Cook: Me parece que el Comité ha hecho un progreso muy significativo en la aplicación del artículo 5.a $)^{5}$, que es la norma sobre estereotipación. Creo que el caso Vertido vs. Filipinas ${ }^{6}$ es quizás uno de los mejores ejemplos de la forma en que el Comité identifica y nombra los estereotipos que actúan en el contexto de un caso de violación. El Comité también ha hecho algunas observaciones finales muy interesantes a partir de los informes de los Estados Partes. En el libro, por ejemplo, nos referimos a las observaciones finales a Tailandia ${ }^{7}$, donde la clase de estereotipos de género que opera en los textos educativos escolares incentiva a las mujeres a

\footnotetext{
"Ellas dan el golpe" en España, "Un equipo muy especial" en Hispanoamérica.

Convención para la Eliminación de Todas las Formas de Discriminación contra la Mujer. Artículo 5.a. "Los Estados Partes tomarán todas las medidas apropiadas para: a) Modificar los patrones socioculturales de conducta de hombres y mujeres, con miras a alcanzar la eliminación de los prejuicios y las prácticas consuetudinarias y de cualquier otra índole que estén basados en la idea de la inferioridad o superioridad de cualquiera de los sexos o en funciones estereotipadas de hombres y mujeres".
}

6 Comité CEDAW. Caso Karen Tayag Vertido vs. Filipinas. Decisión de 16 de julio de 2010. Comunicación No. $18 / 2008$

Comité CEDAW. Consideración de los informes presentados por los Estados partes en virtud del artículo 18 de la Convención sobre la Eliminación de Todas las Formas de Discriminación contra la Mujer. Segundo y tercer informes periódicos de los Estados Partes: Tailandia. UN Doc. CEDAW/CTHA/2-3, 1997, párr.160. 
adoptar roles domésticos en lugar de roles públicos de liderazgo. Como resultado del diálogo y debate que se dio a propósito de las observaciones finales al informe del Estado, ha habido una iniciativa en Tailandia para revisar la estereotipación de género en los textos escolares.

Considero que uno de los retos futuros del Comité es la redacción de una "Recomendación General" sobre estereotipación, que entregue a los Estados mayores lineamientos sobre los estereotipos y cómo reportar sobre ellos. Me parece que una recomendación de este tipo ayudaría mucho en la comprensión y aplicación del artículo 5.a). Algunos puntos que se deberían incluir en la recomendación serían la naturaleza, contenido y alcance de las obligaciones de los Estados parte. Por ejemplo, la obligación de no promulgar ni aplicar leyes que institucionalicen estereotipos de género dañinos, y la obligación de reformar o derogar las leyes que los contienen. Otra obligación que podría especificarse es la de asegurar que las políticas, regulaciones y programas no estén basados en estereotipos de género y, cuando lo estén, modificarlas.

En conclusión, me parece que hay un largo camino por recorrer en la comprensión de los estereotipos y en el desarrollo de una metodología para identificarlos, nombrarlos y erradicarlos, pero de todas formas el Comité ha dado un muy buen punto de partida en esta materia.

Nicole Lacrampette: ¿Existen recomendaciones finales del Comité acerca de la necesidad de abordar la estereotipación de género en la educación legal? Esta es un área en la que hay deficiencias y no sé si es algo que esté en la discusión pública.

Rebecca Cook: No tengo la certeza de que el Comité lo haya abordado. Creo que lo hicieron en las recomendaciones del caso Vertido. Algunos países han desarrollado programas de educación judicial sobre la estereotipación de género y ciertamente sería muy relevante desarrollar programas que logren identificar por qué los jueces desarrollan prejuicios y estereotipos basados en esos prejuicios.

Tal vez se podrían elaborar guías o protocolos ${ }^{8}$ que analicen distintas decisiones judiciales, como la decisión de la Corte de Filipinas que llevó al caso Vertido, en la cual el juez absolvió al acusado basándose en estereotipos sobre las mujeres.

\section{Nicole Lacrampette: El caso Vertido suele considerarse como la principal decisión del Comité en materia de estereotipos de género. ¿Está usted de acuerdo con la decisión del Comité en este caso? Si pudiese rescribir la sentencia para abordar mejor la estereotipación de género en el caso, ¿qué cambios haría?}

Rebecca Cook: Creo que el caso Vertido efectivamente es la principal decisión relativa al tratamiento de la estereotipación de género y definitivamente concuerdo con lo que el Comité dijo acerca de la estereotipación en la sentencia judicial de Filipinas.

Si yo fuese a reescribir la sentencia -es una pregunta compleja- creo que habría clarificado, que es el rol del Comité asegurar que los jueces no basen sus decisiones en estereotipos de género dañinos. Tal vez el Comité podría haber ido más allá en sus recomendaciones, aunque este es un asunto difícil, en términos de elaborar los tipos de remedios que Filipinas podría buscar. En realidad, no sabemos mucho acerca de cómo remediar la aplicación de estereotipos que hacen los jueces, salvo entrenarlos.

Sin perjuicio de ello, el Comité podría haber dicho algo -aunque esto hubiese sido una recomendación demasiado específica y los órganos de derechos humanos intentan ser bastante generales

Lo que se sugiere es la elaboración de un benchbook. Esto es un libro que da un panorama general del procedimiento legal a un juez. Los jueces usan estos libros como guías para ayudarse en la decisión de un caso. 
al recomendar medidas-, tal vez en las conclusiones finales, sobre el próximo informe periódico de Filipinas.

En definitiva, la decisión del caso Vertido es muy significativa y me gustaría referir a mi coautora, Simone Cusack, quien ha hecho un muy buen comentario de este caso en Human Rights Law Review ${ }^{9}$, analizando exhaustivamente la decisión. Ciertamente la jueza de Filipinas, en su argumentación del caso, basó su decisión en estereotipos de género. Ella tenía preconcepciones acerca de las víctimas de violación.

Creo que, en parte, el rol del Comité es educar a la gente acerca de la estereotipación de género, además de nombrar e identificar los estereotipos, la naturaleza del daño que producen y la forma en que se perpetúan.

Nicole Lacrampette: ¿Qué opina acerca del tratamiento de las obligaciones estatales en ese caso? Este es uno de los asuntos que se discuten en el artículo Simone Cusack recién referido.

Rebecca Cook: Como señalan las autoras, el Comité se confundió en el uso del estándar de debida diligencia, pues los jueces son agentes públicos y por lo tanto el punto en el caso no era que el Estado esté obligado a remediar violaciones de derechos cometidas por privados, sino que el Poder Judicial tiene la obligación de asegurar que sus agentes, incluyendo a los jueces, no estereotipen. Entonces, la forma en que el Comité abordó las obligaciones estatales, en relación al sector privado, probablemente fue equivocada, como explican Simone y Alexander en su artículo.

\section{Nicole Lacrampette: ¿Cómo evaluaría las decisiones posteriores del Comité respecto a la iden- tificación de estereotipos?}

Rebecca Cook: Hay una decisión reciente del Comité, en el caso L.C. vs. Perú ${ }^{10}$, en que una adolescente que había sufrido una agresión sexual por parte de privados, intentó suicidarse. El caso se trató de la denegación de cuidado apropiado para tratar las lesiones que ella experimentó tras el intento de suicidio; ese cuidado le fue negado porque estaba embarazada. En este caso, el Comité pudo haber indicado que el Estado de Perú, a través de sus médicos -que son empleados del Estado- trataron a L.C. como un instrumento reproductivo, sin consideración de sus necesidades de atención de salud como paciente. Pero el Comité no se refirió a este punto y tampoco se refirió al tratamiento del supuesto violador.

Nicole Lacrampette: Además del Comité CEDAW, ¿qué otros órganos internacionales de derechos humanos están avanzando en la identificación de la estereotipación de género como una forma de discriminación?

Rebecca Cook: La Comisión Interamericana de Derechos Humanos (CIDH), en uno de los casos que analizamos en detalle en el libro: María Eugenia Morales de Sierra vs. Guatemala ${ }^{11}$, ha abordado este asunto. Para mi esta es una decisión muy sólida porque hace un excelente trabajo identificando y nombrando los estereotipos. En ese caso, la ley de Guatemala requería, entre otras cosas, que las mujeres obtuvieran el permiso de sus maridos para trabajar fuera del hogar, y establecía que los hombres eran los proveedores principales, es decir, el típico estereotipo de

El artículo referido es el siguiente: CUSACK, Simone y TIMMER, Alexandra. "Gender Stereotyping in Rape Cases: The CEDAW Committee's Decision in Vertido v The Philippines". Human Rights Law Review, No. 11, Vol. No.2, 2011, pp.329-342.

11 CIDH. Caso María Eugenia Morales de Sierra vs. Guatemala. Decisión de 10 de enero de 2001. Informe No.4/01. Caso No.11.625. 
la mujer dueña de casa y el hombre proveedor estaba alojado en la legislación. Y la CIDH hizo un muy buen trabajo en esa decisión.

Más recientemente, la Corte Interamericana (Corte IDH) hizo un buen trabajo en su sentencia de 2009 del caso Campo Algodonero ${ }^{12}$, en la que reconoce los estereotipos que operaban y que impidieron que el sistema de justicia investigara las desapariciones de tres mujeres en Ciudad Juárez. En ese sentido, la Corte IDH hace un buen análisis al reconocer cómo los estereotipos respecto de que las mujeres adolescentes son promiscuas, impidió que la justicia tomara acciones inmediatas para investigar sus desapariciones. Si las hubiesen tomado, tal vez podrían haber salvado las vidas de las tres jóvenes. Entonces, el reconocimiento de la Corte IDH de cómo los estereotipos hostiles se perpetúan en el contexto particular de las autoridades policiales, es realmente visionario. En esa sentencia, la Corte IDH también estableció que se debía realizar capacitación judicial, y me complace decir que la Suprema Corte de México sí cuenta con una unidad de género, donde se capacita a los jueces en esta materia.

\section{Nicole Lacrampette: ¿Conoce algún otro caso en que la decisión pudiese haber sido distinta si el tribunal hubiese tomado en consideración la presencia de estereotipos de género?}

Rebecca Cook: En el libro analizamos una sentencia de Canadá, del caso R. vs. Ewanchuk ${ }^{13}$, en el que un presunto violador fue absuelto por el juez sobre la base de los estereotipos que éste tenía acerca de la mujer que era víctima del caso $^{14}$. Asimismo, hay también una sentencia sudafricana, que comentamos en el libro, del caso Presidente de la República Sudafricana vs. Hugo ${ }^{15}$, en que un hombre intentó obtener su liberación temprana de la prisión a través de un indulto presidencial. Sin embargo, este indulto fue otorgado solo a mujeres que tenían hijos pequeños, pese a que el reclamante también tenía un hijo pequeño que cuidar.

De este modo, hay varias instancias en que los estereotipos operan impidiendo un juicio justo e imparcial.

Por otro lado, hay decisiones en que el reconocimiento de los estereotipos genera muy buenas sentencias. En el mismo caso Ewanchuk, cuando la Suprema Corte de Canadá revocó la decisión del juez inferior, hubo una muy buena opinión concurrente de la magistrada L'HeureuxDubé, que nombró los estereotipos que operaron en el caso.

\section{Nicole Lacrampette: ¿Podría explicar brevemente la metodología que se propone en el libro para categorizar los estereotipos de género?}

Rebecca Cook: Bueno, el primer paso, y lo fundamental, es nombrarlo, y no solo nombrar el estereotipo de género sino que también identificar la clase de estereotipo, por ejemplo, si se trata de un estereotipo de sexo -referido a las capacidades físicas o cognitivas de las mujeres- o de un estereotipo sobre los roles sexuales -que se refieren a lo que las mujeres deberían ser, por ejemplo, amas de casa- y finalmente, identificamos los estereotipos compuestos, que son aqueIlos que resultan cuando el género intersecta con otros factores, tales como la edad, la raza o la

12 Corte IDH. Caso González y otras ("Campo Algodonero") vs. México. Excepción Preliminar, Fondo, Reparaciones y Costas. Sentencia de 16 de noviembre de 2009. Serie C No.205.

13 Suprema Corte de Justicia de Canadá. Caso R. vs. Ewanchuk. Resolución de 25 de febrero de 1999. Caso No.26493. Resolución de 25 de febrero de 1999.

14 En este caso, el estereotipo operó al estimar la Suprema Corte, que había existido un "consentimiento tácito" por parte de la víctima, al no haberse enfrentado directamente a su agresor.

15 Corte Constitucional Sudafricana. Caso Presidente de la República de Sudáfrica vs. Hugo. Decisión de 18 de abril de 1997. Caso CCT/1196. 
religión, entre otros. Por ejemplo, en Canadá estamos teniendo un debate, en Quebec, acerca de si las mujeres que trabajan en el sector público pueden o no usar prendas que les cubran la cabeza. En ese debate se discute mucho acerca del hecho que la prohibición del uso de estas prendas impacta desproporcionadamente a las mujeres musulmanas, quienes usualmente querrán usarlas. En el libro se discuten diversas formas de estereotipos compuestos.

En segundo lugar, es importante identificar también la naturaleza del estereotipo. Por ejemplo, un estereotipo descriptivo podría afirmar que la mayoría de las mujeres son físicamente más débiles que los hombres, pero algunas mujeres son más fuertes que algunos hombres. En este caso, el estereotipo no es necesariamente perjudicial para las mujeres, pero se tornará perjudicial si es usado para negar un puesto de trabajo determinado a una mujer, como sería el caso en que se rechaza contratar a una mujer para una posición como bombero, basando la decisión en que los bomberos deben ser los suficientemente fuertes como para rescatar a una persona de un incendio y las mujeres no lo son, con independencia de las capacidades particulares de la mujer que postula al trabajo.

También es posible identificar estereotipos prescriptivos, que disponen -en las leyes, las políticas públicas y las prácticas sociales- lo que las mujeres deben ser. Es decir, son aquellos que no simplemente describen que muchas mujeres son dueñas de casa, sino que prescriben que las mujeres deben serlo. Finalmente, existen estereotipos falsos, que asignan características falsas a las mujeres. Por ejemplo, el estereotipo de que las mujeres son promiscuas suele utilizarse para difamar a las mujeres. En consecuencia, los estereotipos prescriptivos y los falsos son los más problemáticos cuando se encuentran insertos en las leyes.

Nicole Lacrampette: Pensando en uno de los principales problemas que actualmente afectan a las mujeres latinoamericanas, ¿qué rol puede jugar la comprensión de la estereotipación de género en la superación de las barreras que obstaculizan el acceso de las mujeres a la salud sexual y reproductiva?

Rebecca Cook: Se necesita mucho trabajo en esta área, pues la identificación de los estereotipos de género que operan en el área de la salud está recién en sus inicios. Se han escrito algunos artículos sobre esta materia, pero se requiere mucho más desarrollo. Para comenzar, en el área de la salud, operan estereotipos acerca de la capacidad de las mujeres para tomar decisiones médicas. En ocasiones, los servicios de salud consideran a las mujeres como incapaces para tomar decisiones y pueden requerir que sus padres o parejas otorguen consentimiento en su lugar. Otro estereotipo que opera para negar a las mujeres la capacidad de tomar decisiones médicas, es el de considerar a las mujeres como irracionales o emocionales, lo que las hace necesitar de ayuda para la adopción de decisiones. De aquí tal vez se deriva el estereotipo de que las mujeres son vulnerables, y por ello el legislador debe decidir por ellas, por ejemplo, en el acceso a servicios de aborto.

Otro estereotipo que opera en este sector, relacionado con los anteriores, es de la incapacidad de las mujeres para tomar decisiones morales. Esta es también la razón por la cual, por ejemplo, las mujeres no pueden ser sacerdotes y la autoridad moral solo se concibe en los hombres.

Me parece que una decisión muy destacada en Latinoamérica, que realmente reconoció los estereotipos en juego, es la sentencia de la Corte Constitucional colombiana de 2006 que declaró la inconstitucionalidad de la penalización del aborto ${ }^{16}$. Este fallo identificó el estereotipo imbuido en el Código Penal en razón del cual se penalizaba el aborto en prácticamente cualquier circunstancia, al declarar que no se puede ignorar que las mujeres tienen derecho a la dignidad

16 Corte Constitucional de Colombia. Sentencia de 10 de mayo de 2006. C-355/06. 
y deben ser tratadas de manera acorde a esa calidad, en oposición a ser tratadas como un instrumento reproductivo para la humanidad. La Corte hizo un estupendo trabajo al identificar el estereotipo de las mujeres como instrumento reproductivo que estaba inserto en el Código Penal.

Nicole Lacrampette: Por último, ¿cuáles considera que son las dificultades que podrían encontrar quienes se propongan comenzar a trabajar la identificación de estereotipos de género en los casos de discriminación a nivel nacional?

Rebecca Cook: Me parece que es muy importante trabajar con psicólogos sociales en esta materia, pues la estereotipación de género es un área en la que se está avanzando muy rápido en esa disciplina y es importante que los abogados trabajen de manera interdisciplinaria para comprender la naturaleza de la estereotipación y la forma en que resulta dañina, ya que los abogados no tenemos las herramientas para llegar a descubrir la naturaleza de la estereotipación.

También hay un amplio desarrollo de literatura acerca de los prejuicios, que puede resultar útil para este tema. El prejuicio se refiere a la forma en que prejuzgamos las cosas a partir de estereotipos, de manera que el trabajar esta materia en conjunto con la psicología social también podría ser muy útil. 